\title{
Outcome of Fixed Dose of Radioiodine Therapy in Hyperthyroid Patients at NINMAS
}

\author{
Sharmin Quddus, Fatima Begum, Nasreen Sultana, Rahima Perveen, Tapati Mandal, Urnas Islam \\ Mohammad Simoon Salekin, Sadia Sultana and Nurun Nahar \\ National Institute of Nuclear Medicine and Allied Sciences (NINMAS), Dhaka, Bangladesh
}

Correspondence Address: Sharmin Quddus, Principal Medical Officer, National Institute of Nuclear Medicine and Allied Sciences, Block-D, 7th-10th floor, BSMMU Campus, Dhaka-1000, Bangladesh, e-mail:sharmininm@gmail.com

\begin{abstract}
Objective: The modified fixed doses of radioactive iodine (RAI) in different types of hyperthyroidism had been practiced at National Institute of Nuclear Medicine \& Allied Science (NINMAS) according to Society of Nuclear Medicine Bangladesh (SNMB) protocol since 2002 which was upgraded in 2015. The objective of the study was to observe the treatment outcome in modified fixed dose on previous protocol.

Patients and Methods: In the present study the outcome of radioiodine therapy of hyperthyroid patients was retrospectively evaluated in 1349 consecutive primary hyperthyroid patients treated from January 2010 to December 2014 at NINMAS. Diagnosis of hyperthyroidism was done by thyroid function test; thyroid stimulating hormone (TSH), free triiodothyronine (FT3) \& free thyroxine (FT4), 99m Technetium scan, thyroid radioiodine uptake and ultrasound imaging of thyroid gland. All patients received a fixed dose $(8-29 \mathrm{~m} \mathrm{Ci})$ of radioactive iodine (RAI) depending on types of hyperthyroidism, visual assessment of gland size and severity of disease at diagnosis. They were followed up at 2 months of therapy, then every three months intervals for first year and thereafter 6 monthly up to 5 years or as needed during fluctuation of thyroid function.

Results: Among the study population, 832 patients had diffuse toxic goiter (Graves' disease), 369 patients were diagnosed as toxic multinodular goiter and 148 patients with single toxic nodule. At one year follow-up, permanent hypothyroidism occurred in $61.62 \%$ of patients and the cumulative incidence of hypothyroidism progressively increased up to $79.25 \%$ after 5 years. Cure or success of RAI therapy was considered as attainment of euthyroid state or hypothyroid state. About 11.26 $\%$ patients received more than single dose.

Conclusion: Fixed dose RAI therapy is very much cost effective mode of treatment for primary hyperthyroidism with $\sim 89 \%$ success by giving single dose.

Keywords: Hyperthyroidism, hypothyroidism, radioactive iodine.
\end{abstract}

\section{INTRODUCTION}

Hyperthyroidism is a common endocrine disorder worldwide with prevalence of 2:1000 in iodine sufficient area (1). The modes of treatment available are antithyroid drugs, surgery and radioiodine therapy (1). At NINMAS, Radioactive iodine (RAI) therapy by 131I for hyperthyroidism was first introduced in 1980. Initially for long years calculated dose of RAI was used to treat the primary hyperthyroid calculating thyroid gland size and radio-iodine uptake. Then a protocol was developed in 2002 on fixed dose of RAI therapy through a national workshop on the basis of consensus of nuclear medicine specialists, endocrinologists, medicine specialist and ENT surgeons. Since then the fixed dose RAI therapy had been practiced at thyroid division, NINMAS. The guidelines were upgraded in 2015(2).

The objectives of this study was to evaluate the fixed dose RAI therapy in hyperthyroid patients referred to NINMAS according to guidelines for hyperthyroid patients by the Society of Nuclear Medicine, Bangladesh (3). In this study, a total 1349 hyperthyroid patients were evaluated at different time period of follow up from 1 to 5 years after fixed dose RAI.

\section{PATIENTS AND METHODS}

This retrospective study, carried out at the NINMAS, Bangabandhu Sheikh Mujib Medical University (BSMMU), Dhaka where patients with primary hyperthyroidism were referred by the physicians for radioiodine treatment. The study period was from January 2010 to December 2014. Total 1376 cases received fixed dose RAI therapy during the mentioned time period. Out of them, $27 \mathrm{did}$ not complete follow-up criteria therefore, not included for final analysis. Remaining 1349 hyperthyroid patients treated with radioiodine were analyzed.

After thorough physical examination, radio-active iodine uptake test, $99 \mathrm{mTc}$ thyroid scan, biochemical 
tests like free tri iodothyronin (FT3), free thyroxine (FT4) and thyroid stimulating hormone (TSH) level and ultrasound imaging of thyroid gland for determination of the type of hyperthyroidism either diffuse toxic goiter or toxic nodular goiter and also for the size of the gland. Size of the gland was considered to be an important factor to evaluate fixed dose of the radioiodine therapy for subjective assumption. Then the patient was prepared for the first dose of radioiodine therapy. In this study, antithyroid medication was given to all patients 2 days prior and after radioiodine therapy upto one month.

According to Society of Nuclear Medicine Bangladesh (SNMB) protocol 2002, the modified fixed doses of radioiodine in different types of hyperthyroidism are as follows: for Graves' disease: 3-15 mCi; for multinodular goiter: $20-30 \mathrm{mCi}$ and for toxic nodule: $15-25 \mathrm{mCi}$ (3). After radio-iodine therapy, patients were first followed up after 2 months, then 3monthly at first year and subsequently 6 monthly up to 5 years. Outcome of radioiodine treatment was considered as success when patients become euthyroid or hypothyroid. Failure of RAI treatment was considered when patients remained hyperthyroid after six months of therapy.

Hyperthyroid state was declared as when FT3, FT4 level were greater than normal and TSH level less than $0.3 \mathrm{mIU} / \mathrm{L}$. In most of the cases increased diffuse accumulation of Tc-99m was seen on thyroid scan and I-131 uptake more than $10 \%$ in 2 hours and more than $25 \%$ in 24 hours were found. Hypothyroid condition was considered when TSH level $>5 \mathrm{mIU} / \mathrm{L}$ and FT3 and FT4 level were less than normal. Patients who developed hypothyroidism were given levothyroxine replacement therapy. In cases of persisting hyperthyroidism, second dose of RAI therapy in higher dose was given after six months. Data were expressed in number and percentages.

\section{RESULTS}

The demographic information of the hyperthyroid patients with the age of the patients, disease profile, number of dose taken, amount of dose intake in treatment and according to sex distribution were shown in Table- 1 and Table 2 showed treatment outcome.

Table 1: Demographic Information of Patients (n =1349)

\begin{tabular}{|c|c|c|}
\hline & $\mathrm{n}(\%)=556(41.2)$ & $\begin{array}{l}\text { Female } \\
\mathrm{n}(\%)=793(58.8)\end{array}$ \\
\hline \multicolumn{3}{|l|}{ Age categories } \\
\hline $18-30$ years & $112(20.1)$ & $264(33.3)$ \\
\hline $31-40$ years & $129(23.2)$ & $190(24.0)$ \\
\hline $41-50$ years & $131(23.6)$ & $185(23.3)$ \\
\hline $51-60$ ycars & $83(14.9)$ & $112(14.1)$ \\
\hline$>60$ ycars & $101(18.2)$ & $42(5.3)$ \\
\hline \multicolumn{3}{|l|}{ Discase Profile } \\
\hline $\begin{array}{l}\text { Diffuse Toxic goiter } \\
\text { (Graves' Disease) }\end{array}$ & $403(72.5)$ & $429(54.1)$ \\
\hline Toxic Multinodular goiter & $83(14.9)$ & $286(36.1)$ \\
\hline Single Toxic nodule & $70(12.6)$ & $78(9.8)$ \\
\hline \multicolumn{3}{|l|}{ Number of dose given } \\
\hline Single dose & $510(91.7)$ & 697 (87.9) \\
\hline Second dose & $28(5.0)$ & $53(6.6)$ \\
\hline Third dose & $13(2.3)$ & $26(3.2)$ \\
\hline Fourth dosc & $3(0.5)$ & $8(1.0)$ \\
\hline Fifth dose & $2(0.35)$ & $5(0.6)$ \\
\hline \multicolumn{3}{|l|}{$\begin{array}{l}\text { Amount of Dosc } \\
\text { administered }\end{array}$} \\
\hline $8-10 \mathrm{mCi}$ & $280(50.4)$ & $472(59.5)$ \\
\hline $11-15 \mathrm{mCi}$ & $246(44.24)$ & $225(32.40)$ \\
\hline $15-20 \mathrm{mCi}$ & $24(2.3)$ & $58(7.3)$ \\
\hline $21-25 \mathrm{mCi}$ & $03(0.5)$ & $2(0.25)$ \\
\hline $26-29 \mathrm{mCi}$ & $03(0.5)$ & $4(0.50)$ \\
\hline
\end{tabular}

Table 2: Treatment outcome after 3, 6 and 12 months $($ Total $=1349)$

\begin{tabular}{llll}
\hline Time interval & Euthyroid (\%) & Hypothyroid (\%) & Hyperthyroid (\%) \\
\hline & $736(54.55)$ & $38(28.24)$ & $232(17.19)$ \\
6 months & $512(37.95)$ & $672(49.81)$ & $165(12.23)$ \\
12 months & $363(26.90)$ & $834(61.62)$ & $152(11.26)$ \\
\hline
\end{tabular}

Outcome of RAI therapy was analyzed at 5 years follow-up shown in Table 3. All the diffuse toxic goiter patients become hypothyroid after 5 years. Among the total toxic nodular patients (517), 280 (54\%) patients remained in euthyroid state and 237 (46\%) patients were in hypothyroid state. Total 152 patients remained in hyperthyroid state after one year of follow up which may be due to persistent of 
hyperthyroidism or recurrence of disease. Among them 138 patients received multiple doses of RAI and rest 14 patients denied to receive second dose and continued anti-thyroid drugs (Table $3 \&$ Table 4 )

Table 3: Treatment outcome after 5 years $($ Total $=1349)$.

\begin{tabular}{llll}
\hline $\begin{array}{l}\text { Treatment } \\
\text { Outcome }\end{array}$ & Malc & Femalc & Total \\
& $\mathrm{n}(\%)=556(41.2)$ & $\mathrm{n}(\%)=793(58.8)$ & $\mathrm{n}(\%)=1349(100)$ \\
\hline Hypothyroid & $436(32.32)$ & $633(46.92)$ & $1069(79.25)$ \\
Euthyroid & $94(6.9)$ & $186(13.78)$ & $280(20.75)$ \\
\hline
\end{tabular}

Table 4: Dose requirement in patients according to disease categories $(n=1349)$

\begin{tabular}{|c|c|c|c|c|}
\hline \multirow{4}{*}{ Variables } & \multicolumn{2}{|c|}{$\mathrm{n}(\%)=1211(89.77)$} & \multicolumn{2}{|c|}{$\begin{array}{l}\text { Multiple dose } \\
\mathrm{n}(\%)=138(10.22)\end{array}$} \\
\hline & Male & Female & Male & Female \\
\hline & $\mathrm{n}(\%)$ & $\mathrm{n}(\%)$ & $\mathrm{n}(\%)$ & $\mathrm{n}(\%)$ \\
\hline & $504(41.61)$ & $707(58.38)$ & $42(31.81)$ & $96(69.56)$ \\
\hline $\begin{array}{l}\text { Diffuse Toxic goiter } \\
\text { (Graves' Disease) }\end{array}$ & $379(31.4)$ & $369(30.6)$ & $19(13.76)$ & $59(42.75)$ \\
\hline $\begin{array}{l}\text { Toxic multinodular } \\
\text { goiter }\end{array}$ & $61(5.1)$ & $266(21.96)$ & $23(16.66)$ & $31(22.46)$ \\
\hline Single Toxic Nodule & $64(5.2)$ & $72(6.0)$ & $0(0.0)$ & $6(4.2)$ \\
\hline
\end{tabular}

\section{DISCUSSION}

Radioiodine therapy for hyperthyroidism was first introduced at our institute in the year of 1980. Medical records of all patients after RAI therapy had been archived and followed up by thyroid division of this institute. Initially calculated dose of RAI was used and fixed dose RAI therapy had been practiced since 2002. In the present study, we analyzed outcome of fixed dose RAI therapy in 1349 primary hyperthyroid patients. Other authors also mentioned that the fixed dose method is simpler and more cost effective for Graves' disease, toxic multinodular goiter and toxic adenoma (4).

In this study, diffuse toxic goiter (Graves' disease) was found the commonest cause followed by toxic multinodular goiters which are comparable to other reported studies $(3,5)$. Female predominance of hyperthyroidism was noticed in the presented study which was similar to other studies (5).

This institute is a tertiary referral hospital. Patients are referred to this institute from all over the country. We chose fixed and comparatively higher dose for all the patients because the cure rate is > 90\% whereas low dose is more likely to cause treatment failure (6). Persistent or recurrent hyperthyroidism after radioiodine treatment is not uncommon and it usually required re-treatment. Failure of RAI therapy was defined as receipt of additional doses of radioiodine. Successful or failed treatment of hyperthyroidism was evaluated on thyroid function studies at defined time periods after radioiodine treatment. Resistant cases after first dose of radioactive treatment were seen in 152 patients (11.26\%), who remained hyperthyroid six months after treatment. Second dose of radioiodine therapy could be given in 138 patients and rest 14 patients were on antithyroid drugs. After second dose, 31 patients $(22.46 \%)$ required 3rd dose and 39 patients $(28.26 \%)$ required more than three doses.

In other studies, persistent or recurrent hyperthyroidism occurs in $21 \%-29 \%$ of patients treated with conventional doses of radioactive iodine (7). Lower persistent or recurrent hyperthyroidism rates are achievable with higher doses of radioiodine therapy. In other hand, higher treatment doses increase the adverse radiation effects. The persistent hyperthyroidism after I-131 therapy is still a therapeutic problem. Multiple doses of RAI resulted to $100 \%$ cure of hyperthyroidism but in the majority of these patients, hypothyroidism is the resulting adverse effect (8). Hyperthyroidism was treated at the cost of hypothyroidism. Hypothyroidism is acceptable than hyperthyroidism because it is easy to treat under physician's control. In our study the persistent hyperthyroidism is higher in diffuse toxic goiter than in multinodular goiter or single toxic nodular goiter resulting $56.52 \%, 39.13 \%, 4.34 \%$ 
These results are also similar to the data reported by the earlier mentioned author (8).

Antithyroid drugs have been reported to modify the response to radio-iodine through an ill-defined mechanism of radiation resistance. It has also been observed that antithyroid drugs started within 8 days after radioiodine treatment produce less frequent hypothyroidism but a reciprocal increase in persistent hyperthyroidism (9). In this study, we have given antithyroid medication to all our patients 2 days prior and after radioiodine therapy upto one month.

In a study by Allahabadia et al. (10) which included 813 patients, the authors found that male patients with large goiter are more likely not to respond to single dose of 131I even with doses of $370 \mathrm{MBq}$. Male patients had a significantly worse outcome after treatment than females, despite a reduced prevalence of palpable goiter in males. This observation was even more marked in those with Grave's disease, where the cure rate with a single dose of radioiodine was only $50 \%$ (10). But this finding is discordant with our results. In present study, female predominance $(62.31 \%)$ was found in treatment failure group.

Limitations of this study are retrospective nature of study. The factors responsible for radiation resistant to thyroid gland are not analyzed in this study.

\section{CONCLUSION}

It can be concluded from the results of modified fixed dose RAI therapy of the present study that it is a costeffective and simple mode of treatment in the three types of primary hyperthyroidism. Outcome of RAI treatment came as hypothyroid state $79.25 \%$ and euthyoid state $20.75 \%$ after 5 years of RAI therapy. Thus success rate was considered $100 \%$ when euthyroidism as well as hypothyroidism were encountered as cure of the hyperthyroid state. Multiple doses of RAI in hyperthyroid patients were needed in $11.26 \%$ cases after six months of RAI therapy. Toxic nodular goiter patients remained in euthyroid state in higher number showing $54 \%$ at five years.

\section{REFERENCES}

1. Douglas S, Ross MD. Radioiodine Therapy for Hyperthyroidism. N Engl J Med 2011; 364: 542-50.

2. Begum F, Sultana S, Nahar $N$ et al. Management of Hyperthyroidism by Radioactive Iodine (RAIT)-SNMB Guidelines. BJNM 2015 Jan;18:85-88.

3. Alam F, Islam M ASM, Karim MA. Therapy protocol for Thyroid Carcinoma and Thyrotoxicosis with I-131 Adopted in the National Workshop on Management of Thyroid Cancer \& Thyrotoxicosis by Nuclear Medicine Technique-A Consensus Report. Bangladesh Journal of Nuclear Medicine July 2002; 5:39-42.

4. Khalid Y, Barton D M, Baskar et al. Efficacy of Fixed High Dose Radioiodine Therapy for Hyperthyroidism - a 14 year Experience: A focus on Influence of Pre-treatment Factors on Outcomes. BJMP 2011; 4 (3):A 435.

5. Lewis A, Atkinson B, Bell P et al. Outcome of I-131 Therapy in Hyperthyroidism using a $550 \mathrm{MBq}$ Fixed Dose Regimen. Ulster Med J. 2013 May; 82(2):85-88.

6. Schneider David F, Soderman Philip E, Jones Michaela F et al. Failure of Radioactive iodine in Treatment of Hyperthyroidism. ArsSurg Oncol.2014 December;21(13):4174-80.

7. Manohar K, Mittal B R, BhoilA et al. Factors Predicting Treatment Failure in Patients Treated with Iodine-131 for Grave's Disease. WJNM. 2013 May-Aug; 12(2):57-60.

8. Knapska-Kucharska M, Oszukowska L, Lewinski A. Analysis of Demographic and Clinical Factors Affecting the Outcome of Radioiodine therapy in patients with Hyperthyroidism. Arch Med Sci 2010; 6, 4:611-616.

9. Sztal-Mazer S, Nakatani V Y,Bortobni L G et al. Evidence for Higher Success Rates and Successful Treatment Earlier in Grave's Disease with Higher Radioactive Iodine Doses. Thyroid 2012;22(10):991-94.

10. Allahabaida A, Daykin J, Sheppard M et al. Radioiodine Treatment of Hyperthyroidism-Prognostic Factors for Outcome. Journal of Clinical Endocrinology and Metabolism 2001 August;86(8):3611-7. 